\title{
Monte Carlo simulation of kilovolt electron transport in solids
}

\author{
J. D. Martinez, R. Mayol, and F. Salvat \\ Facultat de Fisica (ECM), Universitat de Barcelona. Diagonal 647, E 08028 Barcelona, Spain
}

(Received 19 December 1988; accepted for publication 20 November 1989)

\begin{abstract}
A Monte Carlo procedure to simulate the penetration and energy loss of low-energy electron beams through solids is presented. Elastic collisions are described by using the method of partial waves for the screened Coulomb field of the nucleus. The atomic charge density is approximated by an analytical expression with parameters determined from the DiracHartree-Fock-Slater self-consistent density obtained under Wigner-Seitz boundary conditions in order to account for solid-state effects; exchange effects are also accourted for by an energydependent local correction. Elastic differential cross sections are then easily computed by combining the WKB and Born approximations to evaluate the phase shifts. Inelastic collisions are treated on the basis of a generalized oscillator strength model which gives inelastic mean free paths and stopping powers in good agreement with experimentai data. This scattering model is accurate in the energy range from a few hundred $\mathrm{eV}$ up to about $50 \mathrm{keV}$. The reliability of the simulation method is analyzed by comparing simulation results and experimental data from backscattering and transmission measurements.
\end{abstract}

\section{MTRODUCTION}

A quantitative description of multiple scattering processes suffered by electrons moving in solids is of basic importance in many experimental situations. In particular, such a description is required for quantitative analysis in Auger electron spectroscopy ${ }^{\prime} \mathrm{x}$-ray phoroelectron spectros$\mathrm{copy}^{2}{ }^{2}$ and conversion electron Mössbauer spectroseopy. ${ }^{3}$ The major aim of the present work is to describe a comparatively simple scattering model suitable for Monte Carlo (MC) simulation of multiple scattering of electrons with kinetic energies of up to several tens of $\mathrm{keV}$.

Elastic scattering in MC simulations has usually been described through the screened Rutherford cross section, i.e., the first Born approximation for an exponentially screened Coulomb field. ${ }^{4-7}$ The limitations of such a description arise from the failure of the Born approximation at low energies and from the differences between the exponentially screened field and the real one. For moderately low energies, one must rely on a realistic description of the atomic charge distribution and on a partial wave treatment to derive suffciently accurate differential cross sections (DCS). Purely numerical partial wave calculations have been occasionally used in MC simulations ${ }^{1,8-10}$

It should be noticed that the scattering potential for an atom in a condensed material differs from that of the isolated atom. Solid-state effects have been approximately taken into account by Green and Leckey ${ }^{15}$ in the case of aluminum. The scattering field proposed by these authors is obtained as a sum of contributions from neighboring lattice sites. An alternative and more systematic procedure is to tise the atomic potential obtained from self-consistent atemic structure calculations under Wigner-Seitz boundary conditions. This approach has been recently used by Liliequist et al. ${ }^{12}$

In the present work we use an analytical scattering field with parameters obtained by fitting the self-consistent atomic density computed under Wigner-Seitz boundary condiions. The DCS are evaluated by the method of partial waves with this analytical field. The numerical effort to evaluate the phase shifts is largely reduced by using approximate calculation methods which have been shown to give accurate DCS for electron energies above about $100 \mathrm{eV}$ and isolated atoms. ${ }^{13}$

Much work has been devoted to developing simple inelastic scattering models for use in MC simulations of electron transport. A number of the proposed nodels ${ }^{4-6,14,15}$ are based on the continuous slowing down approximation (CSDA), i.e., the electrons are assumed to lose energy continuously according to the Bethe stopping power formula. As the CSDA fails to reproduce energy straggling effects (see, however, Refs. 5, 14-16), it is useful only in those situations where the details of the energy spectrum are not required (for instance, in computations of backscattered fractions for beams of moderately high energy).

Attempts have also been made to incorporate detailed inelastic models into $M C$ simulations. In the detailed procedures, the energy loss in single inelastic collisions is determined from suitable differential cross sections (DCS) corresponding to the different inelastic mechanisms. Of course, the reliability of the detailed $\mathrm{MC}$ simulations relies upon the accuracy of the adopted DCS. Gryzinski's ${ }^{17}$ semiclassical DCS have been repeatedly used ${ }^{7,8,18}$ to simulate bound shell ionizations; conduction-band excitations have occasionally been described through the dielectric response function. ${ }^{19,20}$

Recently, generalized oscillator strength (GOS) models have been proposed leading to simple analytical (although approximate) expressions for the mean free path and stopping power in fairly good agreement with the available experimental data. ${ }^{21-23}$ In this work, we present a similar GOS model which introduces a more accurate description of the conduction-band excitations and approximately accounts for exchange effects and low-energy corrections to the first Born approximation.

The adopted scattering model is described in Sec. II. We begin with an outline of the elastic DCSs and consider in detail the evaluation of the DCSs for inelastic processes. Inelastic mean free paths and stopping power of electrons in aluminum and gold computed from these DCSs are present- 
ed in Sec. III and are shown to be in fairly good agreement with experimental data and other theoretical calculations. In Sec. IV we describe the simulation algorithm implementing the present scattering model. Simulation results for backscattering and transmission geometries are presented in Sec. $V$. The comparison of these results with experimental data indicates that the proposed model and the $\mathrm{MC}$ algorithm provide reliable results for electron energies in the interval from a few hundred $\mathrm{eV}$ to about $100 \mathrm{keV}$.

\section{H. SCATTERING MODEL}

In this section we consider the DCSs for the different interactions of electrons with kinetic energy $E$ within the sample. We use atomic Hartree units $\left(\hbar=m_{e}=e=1\right)$ unless otherwise specified. For the sake of brevity, we shall consider only single-element solids.

\section{A. Elastic scattering}

Elastic collisions of moderately fast electrons with a target atom can be described by using the theory of scattering by a center of forces. ${ }^{24}$ We shall adopt atomic densities derived from the self-consistent Dirac-Hartree-Fock-Slater (DHFS) method with Wigner-Seitz (WS) boundary conditions to account for solid-state effects. In order to facilitate the incorporation of the elastic DCS into a MC program, it is convenient to approximate the (spherically symmetrical) atomic electron density by a suitable analytical expression. We use the following parameterization of the DHFS density:

$$
\rho(r)=\frac{Z}{4 \pi r} \sum A_{i} \alpha_{i}^{2} \exp \left(-\alpha_{i} r\right), \quad \sum A_{i}=1,
$$

where $Z$ is the atomic number of the target, and $A_{i}$ and $\alpha_{i}$ are parameters which have been given for some WS atoms ${ }^{14}$ and for free atoms $(Z=1-92)$. $^{25}$ It may be noticed that, for WS atoms, the DHFS censity drops abruptly to zero outside the WS sphere whereas the analytical density [Eq. (1)] has a small exponential tail in this region. This difference only affects the DCS at rather small scatiering angles and its effect on multiple scattering is fairly small.

For electron energies below a few $\mathrm{keV}$ (depending on the atomic number of the target), exchange effects with the target electrons will become appreciable. We shall use the local exchange potential of Furness and McCarthy as desoribed in detail in Ref. 13. Charge cloud polarization is known to be noticeable for low electron energies; its influence on the DCS is reduced to an extra contribution which, for energies above $100 \mathrm{eV}$, is negligible except for small scattering angles; therefore, its effect on multiple scattering processes is expected to be comparatively small.

The elastic DCS for collisions with a polar scattering angle between $\theta$ and $\theta+d \theta, d \sigma^{(e)} / d \theta$, is computed by following the procedure described in Ref. 13, i.e., by the partial wave method with phase shifts obtained either from the WKB or Born approximations. This comparatively simple calculation procedure has been shown to lead to quite accurate DCS for elastic scattering of electrons by free atoms. ${ }^{13}$ Unfortunately, no experimental information on single scattering in solids is avalable. The accuracy of the DCS adopted here will be checked a posteriori by comparing $\mathrm{MC}$ results with available experimental data on transmission and backscattering of electron beams in thin solid layers.

The total elastic cross section is given by

$$
\sigma^{\mathrm{e} \mathrm{e}}=\int_{0}^{\pi} \frac{d \sigma^{(\mathrm{e})}}{d \theta} d \theta
$$

The elastic mean free path is

$$
\lambda^{(\mathrm{el})}=\left(N \sigma^{(\mathrm{el})}\right)^{\cdots !},
$$

where $N$ is the number of target atoms per unit volume.

\section{Inelastic scattering}

The DCS for inelastic collisions as obtained from the first Born approximation can be written in the form ${ }^{26}$

$$
\frac{d \sigma^{(i n)}}{d W d Q}=\frac{\pi}{E} \frac{1}{W Q} \frac{d f(W, Q)}{d W},
$$

where $W$ is the energy lost by the free electron in the collision and $Q$ is the recoil energy which is related to the momentum transfer ay $Q=q^{2} / 2$. The DCS (4) splits into two factors: The first one is purely kinematical and independent of the target system, whereas the second one, the so-called generalized oscillator strength (GOS), df $(W, Q) / d W$, completely characterizes the target. The representation of the GOS as a function of $Q$ and $W$ is known as the Bethe surface and has a fairly well-defined topology. ${ }^{26}$ For large energy losses it is reduced to a ridge over the curve $W=Q$ which corresponds to close binary collisions; for relatively small energy losses the Bethe surface presents a structure which is characteristic of the target system.

We consider the description of inelastic interactions on the basis of an independent particle model so that, in the first Born approximation, only single electron excitations are allowed by the usua selection rules. Thus, the different inelastic processes leading to ionization of different bound shells do not interfere with one another and may be treated separately. According to this assumption, we introduce the following GOS model:

$$
\frac{d f(W, Q)}{d W}=\sum Z_{i} \frac{d g_{i}(W, Q)}{d W}
$$

where the summation runs over all bound shells and the conduction band; $Z_{i}$ is the number of electrons in the $i$ th shell or conduction band. The function $d g_{i}(W, Q) / d W$, which will be referred to as the partial generalized oscillator strength (PGOS), characterizes the excitations of the ith bound shell or conduction band. In order to satisfy the Bethe sum rule ${ }^{26}$ we require

$$
\int_{0}^{\infty} \frac{d g_{i}(W, Q)}{d W} d W=1 .
$$

It should be noticed that with this model, when using $Z_{i}$ as the effective number of electrons in each shell, we neglect the transfer of oscillator strength from inner to outer shells. ${ }^{27}$

The inelastic DCS per electron in the ith shell or conduction band is

$$
\frac{d \sigma_{i}^{(i n)}}{d W d Q}=\frac{\pi}{E} \frac{1}{W Q} \frac{d g_{i}(W, Q)}{d W} .
$$

Energy and momentum conservation lead to the following 
relation between the energy loss $W$, the recoil energy $Q$, and the polar scattering angle $\theta$ :

$$
Q=E+(E-W)-2[E(E-W)]^{1 / 2} \cos \theta
$$

The DCS per electron for collisions with energy loss between $W$ and $W+d W$ is obtained as

$$
\frac{d \sigma_{i}^{(\mathrm{in})}}{d W}=\int_{Q_{\min }}^{Q_{\max }} \frac{d \sigma_{i}^{(\mathrm{in})}}{d W d Q} d Q
$$

where $Q_{\min }\left(Q_{\max }\right)$ is the minimum (maximum) recoil energy in a collision where the energy loss is $W$, which is given by Eq. (8) with $\cos \theta=1(\cos \theta=-1)$. The total cross section per electron is obtained as the integral of Eq. (9) between the minimum energy loss $W_{\min }$, fixed by the exclusion principle and a maximum loss $W_{\max }$ which will be determined below.

The inelastic cross section per atom, the mean free path, and the stopping power are given by

$\sigma^{(\mathrm{in})}=\sum_{i} Z_{i} \int \frac{d \sigma_{i}^{(\mathrm{in})}}{d W} d W, \quad \lambda^{(\mathrm{in})}=\left(N \sigma^{(\mathrm{in})}\right)^{-1}$

and

$$
S=-\frac{d E}{d s}=N \sum_{i} \mathbb{Z}_{i} \int W \frac{d \sigma_{i}^{(i n)}}{d W} d W
$$

respectively.

\section{Ionization of bound shelis}

The PGOS for the ionization of the ith bound shell is approximated as (see Ref. 22)

$$
\begin{aligned}
\frac{d g_{i}(W, Q)}{d W}= & {\left[\left(1-\frac{W_{e}^{2}}{Q^{2}}\right) \delta(W-Q)\right.} \\
& \left.+\frac{2 W_{e}^{2}}{W^{3}} \Theta(W-Q)\right] \Theta\left(W-W_{e}\right)
\end{aligned}
$$

where $\Theta(x)$ is the step function and $\delta$ is the Dirac delta function. The quantity $W_{e}$ is the minimum energy loss necessary to produce ionization in this shell. We use the experimental ionization energies $U_{i}$ for free atoms and, in accordance with the exclusion principle, we take $W_{e}=U_{i}+E_{F}$ where $E_{F}$ is the Fermi energy of the electrons in the conduction band (see below). The first term in Eq. (12) containing the delta function accounts for optically forbidden excitations (close collisions) whereas the second term corresponds to optically allowed excitations (distant collisions). It can be easily verified that the PGOS (12) satisfies Eq. (6).

The DCS (9) corresponding to the PGOS (12) is given by

$\frac{d \sigma_{i}^{(\mathrm{in})}(E)}{d W}=\left(1-\frac{W_{e}^{2}}{W^{2}}\right) \frac{d \sigma_{R}(E)}{d W}+\frac{\pi}{E} \frac{2 W_{e}^{2}}{W^{4}} \ln \left(\frac{W}{Q_{\text {min }}}\right)^{2}$,

where

$$
\frac{d \sigma_{R}(E)}{d W}=\frac{\pi}{E} \frac{1}{W^{2}}
$$

is the Rutherford cross section for binary collisions with free electrons at rest. For large energy losses, Eq. (13) is simplified to Eq. (14).

Exchange and relativistic effects in close collisions are introduced as outlined in Ref. 22: The Rutherford cross section appearing in Eq. (13) is replaced by the Moller cross section

$$
\begin{aligned}
\frac{d o_{M}(E)}{d W}= & \frac{2 \pi}{v^{2}}\left(\frac{1}{W^{2}}+\frac{1}{(E-W)^{2}}+\frac{1}{\left(E+c^{2}\right)^{2}}\right. \\
& \left.-\frac{\left(E+c^{2}\right)^{2}-E^{2}}{\left(E+c^{2}\right)^{2}} \frac{1}{W(E-W)}\right),
\end{aligned}
$$

where $v$ is the electron velocity. This procedure is similar to the one used in the Bethe theory of the stopping power of electrons. In order to account for the increase in the kinetic energy of the incoming electron in the atomic field we also replace the kinetic energy $E$ on the right-hand side of $E q$. (15) by $E+2 U_{i}{ }^{22}$ Relativistic effects in optically allowed excitations can be approximately accounted for by the introduction of a simple correcting factor in the DCS, namely

$$
f_{r}(E)=\frac{2 E}{v^{2}}=\frac{2\left(E+c^{2}\right)^{2}}{c^{2}\left(E+2 c^{2}\right)},
$$

where $c$ is the speed of light in vacuum. The resulting DCS is

$$
\left.\left.\frac{d \sigma_{i}^{(\mathrm{in})}}{d W}=\frac{d \sigma_{i}^{(\mathrm{in})}}{d W}\right]_{j}+\frac{d \sigma_{i}^{(\mathrm{in})}}{d W}\right]_{a}
$$

where

$$
\left.\frac{d \sigma_{i}^{(\mathrm{in})}}{d W}\right]_{f}=\left(1-\frac{W_{e}^{2}}{W^{2}}\right) \frac{d \sigma_{M}\left(E+2 U_{i}\right)}{d W}
$$

is the contribution of optically forbidden excitations and

$$
\begin{aligned}
\left.\frac{d \sigma_{i}^{(i n)}}{d W}\right]_{a}= & f_{r}\left(E+2 U_{i}\right) \frac{2 \pi}{E+2 U_{i}} \frac{W_{e}^{2}}{W^{4}} \\
& \times \ln \left[\frac{E+2 U_{i}}{W}\left[1+\left(1-\frac{W}{E+2 U_{i}}\right)^{1 / 2}\right]^{2}\right]
\end{aligned}
$$

that of optically allowed excitations.

After each ionizing collision the kinetic energy of the electron is reduced to $E-W$ and a secondary electron with kinetic energy $E_{s}=W-U_{i}$ is ejected. We consider the primary electron as the most energetic one after the collision, therefore the maximum energy loss is $W_{\max }=\left(E+U_{i}\right) / 2$.

According to our PGOS model, the recoil energy $Q \mathrm{co}-$ incides with $W$ for optically forbidden excitations, whereas for optically allowed excitations, $Q$ can take values in the interval $\left(Q_{\min }, W\right)$ with a $Q^{-1}$ distribution [see Eqs. (7) and (12)].

\section{Conduction band excitations}

The free-electron gas model provides a suitable basis to describe the excitations of the conduction band of certain materials (which will be referred to as free-electron-like materials), such as alaminum, with valence electrons distributed nearly uniformly in the volume of the solid in addition to tightly bound electron shells. Excitations of free-electron gases with densities of the order of those corresponding to the conduction band of real solids can be described in terms 
of Lindhard's dielectric function. ${ }^{28-30}$ As the DCSs obtained from this dielectric function cannot be expressed analytical$\mathrm{iy}$, it is convenient to seek a simple PGOS model leading to analytical (although approximate) DCSs which are most suited for simulation purposes.

The electron gas may be characterized by the one electron radius $r_{s}$ which is related to the electron density $n$ through $n=3 /\left(4 \pi r_{s}^{3}\right)$. The plasmon energy $E_{p}$ and the Fermi energy $E_{F}$ are given by $E_{p}^{2}=4 \pi n$ and $E_{F}=\left(3 \pi^{2} n\right)^{2 / 3} / 2$. The plasmon-pole approximation ${ }^{22,31}$ in which the excitation spectrum of the electron gas is replaced by a single mode along the plasma resonance line leads to inelastic mean free path and stopping power in good agreement with those obtained from Lindhard's dielectric function ${ }^{25}$ for electron energies larger than the plasmon energy. However, for $W<E_{p}$ this approximation leads to a vanishing DCS over against the Lindhard DCS which is nearly proportional to $W$ (see Refs. 29,30). In order to reproduce the linear behavior of the Lindhard DCS for low-energy losses, we introduce a PGOS model with two modes, one along the plasma resonance line

$$
W_{Q}^{2}=E_{p}^{2}+2 B Q+Q^{2}
$$

with $B=6 E_{F} / 5$ describing the excitation of collective oscillations in the gas, and a second one along the line $Q=W$, corresponding to electron-hole excitations. Thus we set

$$
\begin{aligned}
\frac{d g_{\mathrm{cb}}(W, Q)}{d W}= & {[1-f(Q)] \delta\left(W-W_{Q}\right) } \\
& +f(Q) \delta(W-Q),
\end{aligned}
$$

where

$$
f(Q)=\min \left(1, \alpha Q^{3}\right)
$$

The parameter $\alpha$ which fixes the slope of the DCS for small energy losses will be determined below.

It is convenient to express the DCS (9) corresponding to the PGOS (21) as the sum of contributions due to electron-hole and plasmon-like excitations:

$$
\left.\left.\frac{d \sigma_{\mathrm{cb}}^{(\mathrm{in})}}{d W}=\frac{d \sigma_{\mathrm{cb}}^{(\mathrm{in})}}{d W}\right]_{p-h}+\frac{d \sigma_{\mathrm{cb}}^{(\mathrm{in})}}{d W}\right]_{p}
$$

We have

$$
\begin{aligned}
\left.\frac{d \sigma_{\mathrm{ch}}^{(\mathrm{in})}}{d W}\right]_{e-h}= & \frac{\pi}{E} \alpha W \Theta\left(W_{c}-W\right) \\
& +\frac{d \sigma_{R}(E)}{d W} \Theta\left(W-W_{c}\right),
\end{aligned}
$$

and

$$
\left.\frac{d \sigma_{\mathrm{cb}}^{(\mathrm{in})}}{d W}\right]_{p}=\frac{\pi}{E} \frac{1-f\left(Q_{0}\right)}{Q_{0}\left(B+Q_{0}\right)}
$$

with $W_{c}=\alpha^{-1 / 3}$ and $Q_{0}=\left(B^{2}+W^{2}-E_{p}^{2}\right)^{1 / 2}-B$. The recoil energy in plasmonlike excitations is related to the energy loss $W$ through $\mathrm{Eq}$. (20). In electron-hole excitations we have $Q=W$ and also the emission of a secondary electron whose kinetic energy is assumed to coincide with $W$.

In the limit of low-energy losses, the DCS [Eq. (25)] vanishes and Eq. (24) agrees exactly with the DCS obtained from Lindhard's dielectric function if we take $e^{29,30}$

$$
\begin{aligned}
\alpha= & \frac{1}{4 E_{F} E_{p}^{2}}\left[\frac{1}{1+2 \chi^{2} / 3}+\frac{1}{\chi\left(1-\chi^{2} / 3\right)^{1 / 2}}\right. \\
& \left.\times \tan ^{-1}\left(\frac{\left(1-\chi^{2} / 3\right)^{1 / 2}}{\chi}\right)\right],
\end{aligned}
$$

where $\chi^{2}=(2 / \pi)\left(6 \pi E_{p}^{2}\right)^{-1 / 3}$. The function on the righthand side differs slightly from that given in Ref. 30 since we use a different approximation for the dielectric function [namely we use Eq. (14) from Ref. 29 instead of the second equation after Eq. (6.14) in Ref. 30].

Exchange and relativistic effects are introduced by following the same approximation as for bound shell excitations, i.e., the right-hand side of Eq. (25) and the first term in the right-hand side of Eq. (24) are multiplied by the relativistic correction (16); moreover, the Rutherford cross section in Eq. (24) is replaced by the Moller cross section (15) and, consequently, the maximum energy loss in electronhole excitations is limited to $W_{\max }=E / 2$.

\section{COMPUTED MEAN FREE PATHS AND STOPPING POWERS}

When applying the PGOS model (12) and (21) to freeelectron-like materials we set $n=N Z_{\mathrm{c} b}$ which corresponds to a free-electron gas of density equal to that of the conduction band (i.e., $N$ times the number $Z_{\mathrm{cb}}$ of free electrons per atom). The inverse inelastic mean free path and stopping power for electrons in aluminum are shown in Figs. 1(a) and 1(b), respectively. These figures also include the theoretical results of Ashley, Tung, and Ritchie ${ }^{32}$ who used a GOS description for bound shell ionization and the dielectric theory for conduction-band excitations. The stopping power computed from the Bethe formula is also shown in Fig. 1(b); it agrees with our model predictions for energies above a few $\mathrm{keV}$, but the Bethe stopping power becomes negative for energies slightly below the mean excitation energy ( $166 \mathrm{eV}$ for Al).

In practice, only a limited class of materials present a conduction band with a nearly free-electron-like excitation spectrum. Optical measurements reveal that the oscillator strength distribution, $d f(W, Q=0) / d W$, of real solids in the region of low-energy losses $(<50 \mathrm{eV})$, have structures which differ considerably from the single delta function $\delta\left(E_{p}-W\right)$ used in our PGOS model. Even the most representative free-electron-like material, namely aluminum, presents damping effects, i.e., the plasma mode has a finite width along the $W$ axis. In spite of these difficulties, the present PGOS model provides a fairly accurate description of the slowing down of electrons with energies above a few tens of $\mathrm{eV}$ in any material provided the parameter $r_{s}$ or the effective electron density is suitably chosen. In general, to determine the value of this parameter it is necessary to resort to experimental data, if available, or to more accurate theoretical calculations. On the basis of the local density approximation (LDA) (see, for instance, the formulation in Ref. 33), as the valence electrons are, in general, not strictly uniformily distributed over the sample volume but to some extent located around the lattice ions, an effective $r_{s}$ value smaller than that for a free-electron gas of density $N Z_{\mathrm{cb}}$ must be expected. 

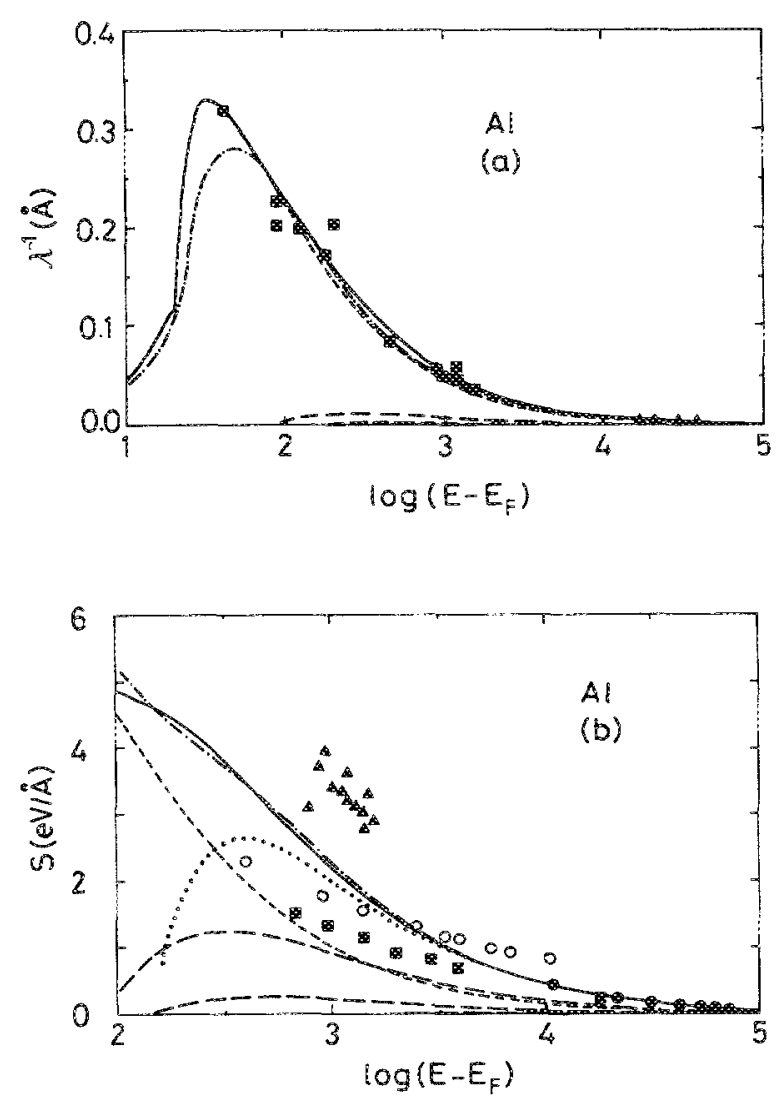

FIG. 1. (a) Inelastic inverse mean free path and (b) stopping power of electrons in aluminum. Energies are given in $\mathrm{eV}$ and referred to the Fermi level. The continuous curves correspond to the results from the present inelastic scattering model. Special symbols are experimental data from different authors quoted in Ref. 32. The theoretical estimates of Ref. 32 are also included (dot-dashed curves). The dotted line in (b) corresponds to the Bethe stopping power. The long-dashed curves are the contributions of the conduction band and bound shells as given by the present model.

The electron inelastic inverse mean free path and stopping power in gold computed from this model and from the LDA are compared with experimental data in Fig. 2. We have taken $r_{s}=0.6 r_{0}$ where $r_{0}=(3 / 4 \pi n)^{1 / 3}$ is the one electron radius corresponding to the average electron density in the conduction band, i.e., $n=N Z_{\mathrm{cb}}$ with $\mathbb{Z}_{\mathrm{cb}}=1 \%$. Our results are in good agreement with the more involved LDA calculations of Tung, Ashley, and Ritchie. ${ }^{33}$ The main advantage of the present model lies in its ability to describe energy straggling, as well as the production of secondary electrons, when used as the basis of MC simulations.

\section{IY. MONTE CARLO PROCEDURE}

The conventional detailed $\mathrm{MC}$ method for simulation of particle transport is used. The results of the simulated experiment, under a given geometry, are inferred from a large number of electron tracks numerically drawn in a computer. Each track is started at a given position, with initial direction and energy in accordance with the characteristics of the source. The state of an electron immediately after a collision (or after entering the sample or starting its trajectory) is characterized by its position r, energy $E$ and director cosines of the direction of fight $d$. The length of the free path to the
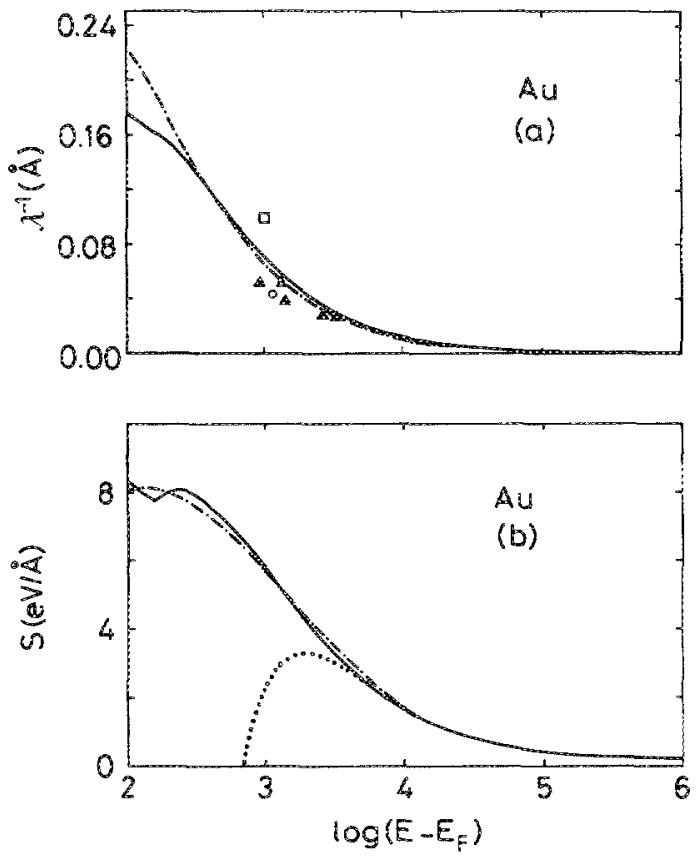

FIG. 2. Inelastic inverse mean free path and stopping power of electrons in gold. The continuous curve corresponds to the present model, the dotdashed line is the LDA result of Tung and co-workers (Ref. 33) and the dotted curve is the Bethe stopping power. Energies are in $\mathrm{eV}$ and are referred to the Fermi level. Special symbols are experimentai data from different authors quoted in Ref. 33 .

next collision, the involved scattering mechanism, the change of direction, and the energy loss in this collision (as well as the initial conditions of the possible secondary electron emitted) are considered to be random variables. The corresponding probability distributions are determined from the DCS of the different scattering processes. Each simulated track is characterized by a series of states $\left\{\mathrm{r}_{n}, E_{n}\right.$, $\left.d_{n}\right\}$, where $\mathrm{r}_{n}$ is the position of the $n$th collision, $E_{n}$ and $\mathbb{d}_{n}$ are the kinetic energy and director cosines of the direction of movement just after that collision.

Let us assume that the electron track has already been simulated to a state $\left\{\mathbf{r}_{n}, E_{n}, \mathbb{d}_{n}\right\}$. The mean free path $\lambda$ between collisions is given by

$$
\lambda^{-1}=N\left(\sigma^{(\mathrm{el})}+\sigma^{(\mathrm{in})}\right) .
$$

The length $s$ of the free path to the next collision is obtained by random sampling from the distribution

$$
p(s)=\lambda^{\cdots 1} \exp (-s / \lambda) .
$$

Thus, the next collision occurs at the position $r_{n+1}$ $=\mathbb{x}_{n}+s d_{n}$. The type of the next collision is decided from the probabilities

$$
p_{I}=\sigma_{I}\left(\sigma^{(\mathrm{el})}+\sigma^{(\mathrm{in})}\right)^{-1}
$$

where the index I runs over the different types of collision, i.e., elastic and different inelastic processes. It is convenient to consider the optically allowed and forbidden excitations (plasmonlike and electron-hole excitations in the case of the conduction band) to be independent processes, characterized by different relations between the energy loss $W$ and the recoil energy $Q$.

If the collision is elastic, the polar scattering angle is 


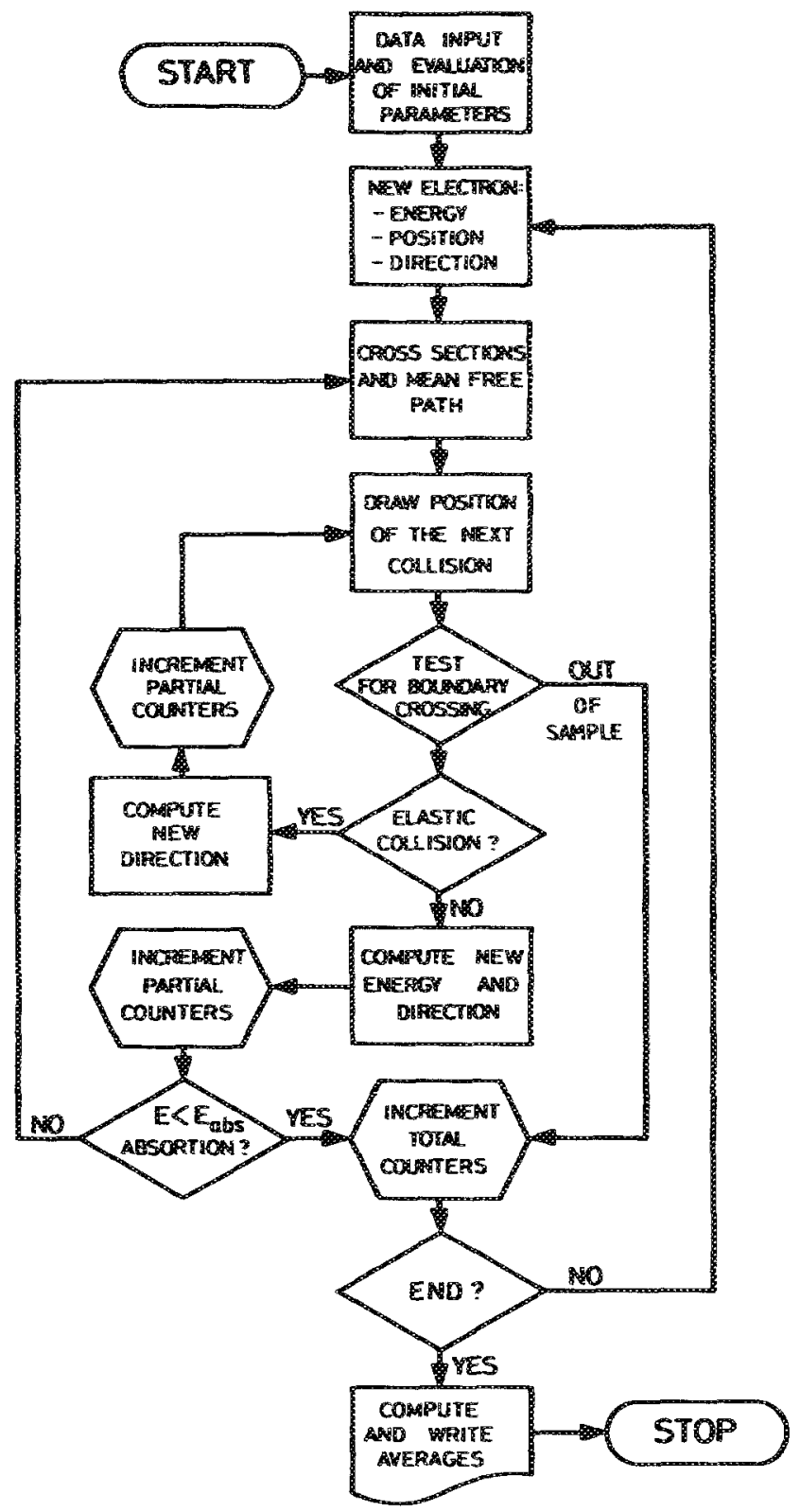

FIG. 3. Flow chart of the Monte Carlo simulation code.

sampled from the distribution

$$
p(\theta)=\frac{1}{\sigma^{(\mathrm{el})}} \frac{d \sigma^{(\mathrm{e})}}{d \theta} .
$$

The azimuthal scattering angle $\varphi$ is sampled uniformly on the interval $(0,2 \pi)$. The new direction cosines $d_{n+1}$ after the collision are obtained by performing a rotation of $d_{n}$ determined by the values of the scattering angles.

If the collision is inelastic, the energy-loss distribution is given by the corresponding DCS normalized to unity. Once the energy loss has been sampled, the energy of the primary electron is reduced, $E_{n+1}=E_{n}-W$, and the recoil energy determined according to the corresponding PGOS. For optically forbidden excitations of bound shells and electron-hole excitation of the conduction band we have $Q=W$-see Eqs. (12) and (21). For optically allowed transitions, $Q$ is sampled according to the $Q^{-1}$ distribution in the interval

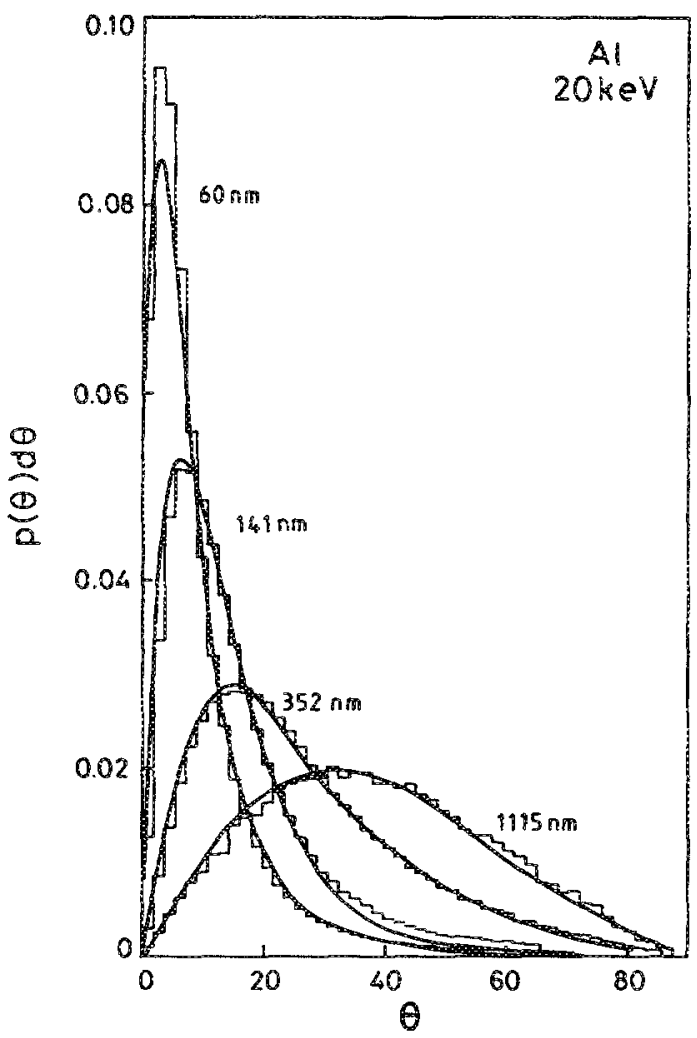

FIG. 4. Angular distributions of $20-\mathrm{keV}$ electrons after traversing an aíminum layer of different thicknesses. The direction of incidence is perpendicular to the layer surface. Experimental data are from Thomas (Ref. 36). Histograms are the results of the present simulations (normalized to the same area as the experimental distributions).

$\left(Q_{\min }, W\right)$, as it follows from Eqs. (7) and (12). For plasmonlike excitations $Q$ is given by Eq. (19).

The polar scattering angle $\theta$ in inelastic collisions is fixed by Eq. (8) and the azimuthal scattering angle is sampled uniformly in the interval $(0,2 \pi)$. The new director cosines $d_{n+1}$ are then determined by rotation of $d_{n}$ as in the elastic case. With the exception of plasmonlike excitations, a secondary electron is emitted at $\boldsymbol{r}_{n+1}$, with initial energy (on

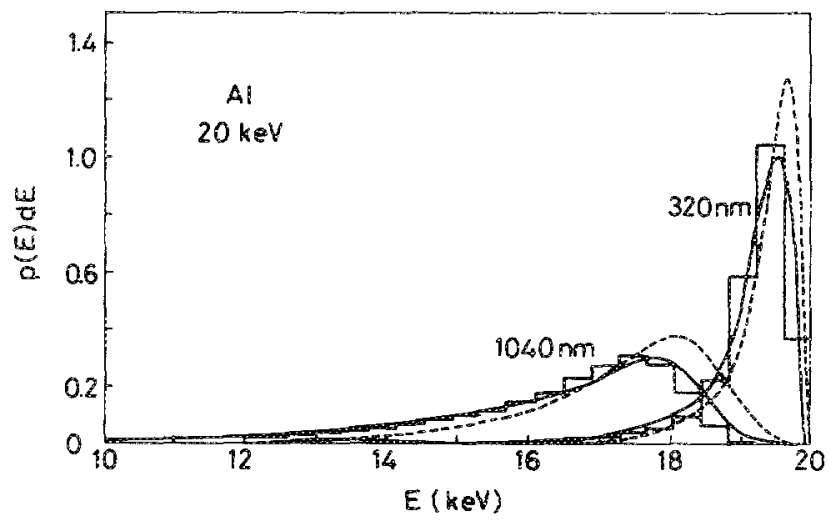

FYG. 5. Energy distributions of $20-\mathrm{keV}$ electrons after traversing an aluminum layer of 320 and $1040 \mathrm{~nm}$. The continuous curves are experimental distributions from Shimizu et al. ( Ref. 5), histograms are the results from the present simulations. The dashed lines are the Monte Carlo simulation results of Ref. 5. All distributions have been normalized to the same area. 

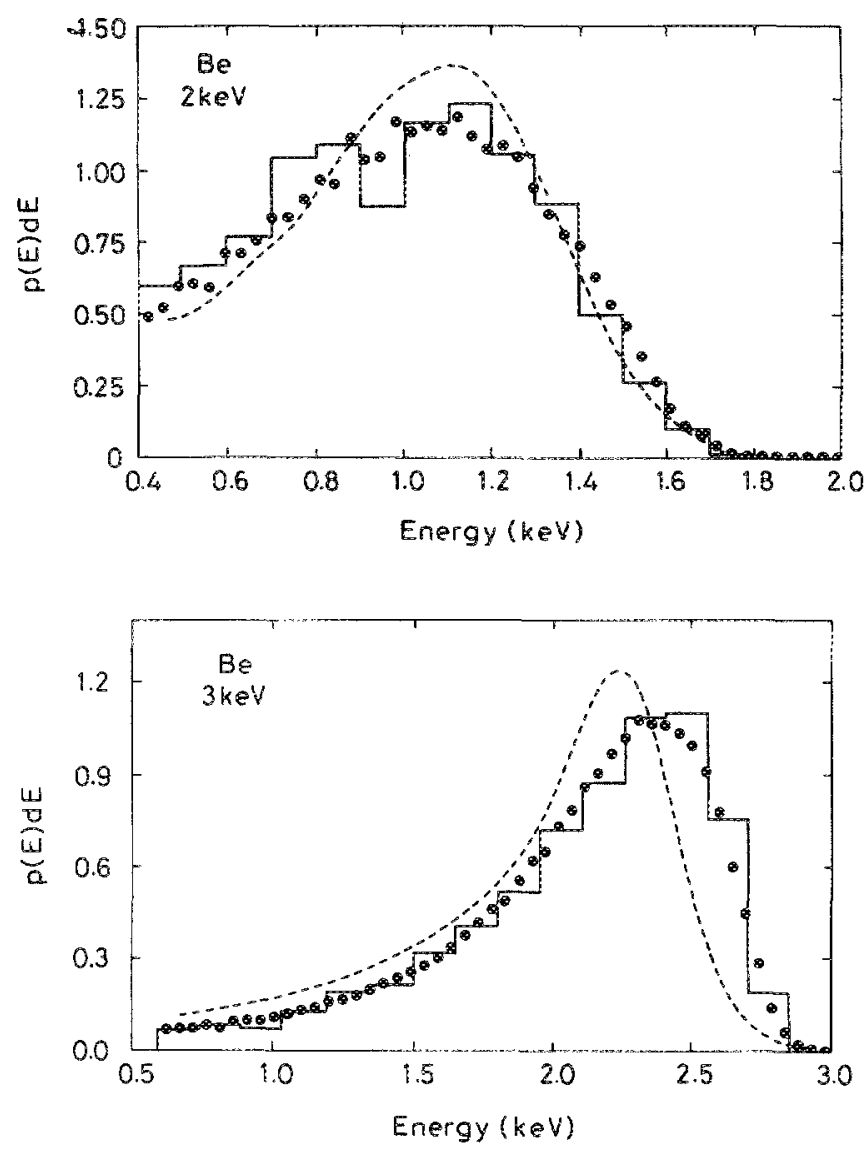

FIG. 6. Energy distributions of 2- and 3-keV electrons transmitted through a beryllium film of $80 \mathrm{~nm}$ at normal incidence. The dashed curves are experimental distributions from Fitting (Ref. 42). The histograms are the results of MC simulations from Liljequist (Ref. 23). The dots are the results of the present simulations. All the distributions have been normalized to the same area.

average) equal to the energy released by the primary (minus the ionization energy for bound shell ionizations). The secondary electron moves perpendicularly to the primary one and over the scattering plane, determined by $r_{n+1}$ and the directions of $d_{n}$ and $d_{n+1}$. The variables defining the initial state of the secondary electron are stored and the trajectory of the primary one is followed up to its end by repeating these steps.

A track is finished either when it crosses a boundary of the sample or when the energy becomes smaller than a given value, $E_{\mathrm{abs}}$. This value corresponds to the energy where electrons are assumed to be effectively stopped and absorbed in the medium ( $=100 \mathrm{eV}$ in the simulations reported below) or to the lower end of the energy interval covered by the detector. After finishing the primary track, secondary tracks must also be simulated from the initial states previously determined. Obviously, only secondaries with an initial energy greater than $E_{\text {abs }}$ must be considered. This makes the number of secondaries per primary track relatively small. Moreover, as the energies of the secondaries are usually small, these are not likely to produce other secondaries with energy larger than $E_{\text {abs }}$.

The flow chart of the simulation program for a homogeneous sample is given in Fig. 3. For the sake of clarity, we
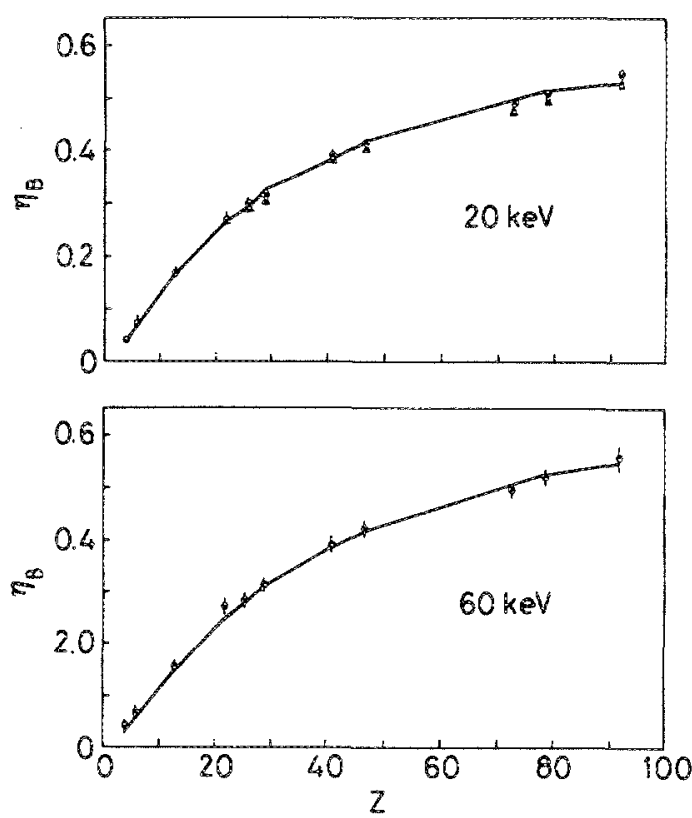

FIG. 7. Backscattered fraction of 20 -and $60-\mathrm{keV}$ electrons impinging normally on thick shabs as a function of the atomic number of the target materia1. The continuous curye corresponds to the experimental measurements of Neubert and Rogaschewski (Ref. 38). Circles are the results of the present simulations, triangles are the simulation results neglecting the contributions of secondary electrons.

have not included the generation of secondary electrons. The simulation of the secondary electrons left by each primary should be performed just before starting the new primary track.

The simulation algorithm was implemented with a FORTRAN code. The random sampling of the different variables was performed by using pseudorandom numbers uniformly distributed in the interval $(0,1)$ obtained from a Tausworthe generator ${ }^{34,35}$ with a period of $2^{250}$. It is worth noticing that this is a value that is large enough to prevent the reinitiation of the random series during a single simulation. The code was run on an BBM 3090/200 computer. The majority of the results presented in the next section were obtained from single runs of $1500 \mathrm{~s}$ involving the generation of some 30000 electron tracks (depending on the geometry

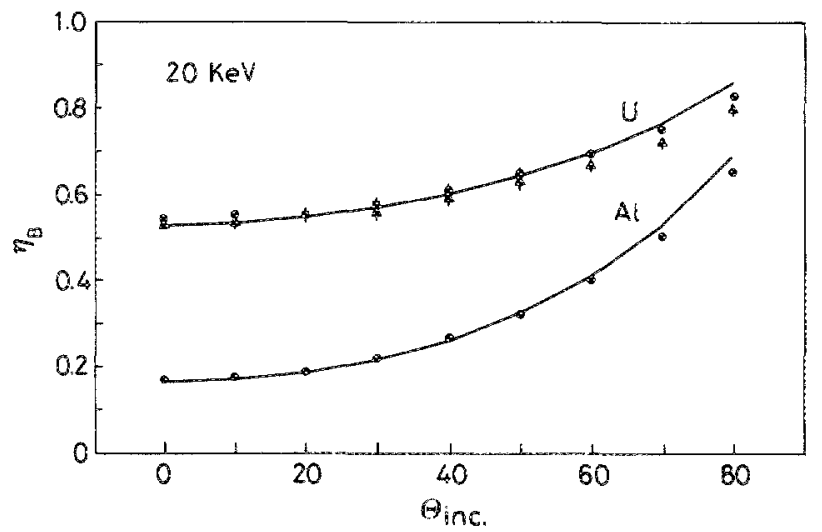

FIG. 8. Backscattered fraction of $20-\mathrm{keV}$ electrons impinging on thick aluminum and uranium slabs as a function of the angle of incidence relative to the surface normal. Details are the same as in Fig. 7. 
and the atomic number of the target).

It should be pointed out that coherent scattering and channeling are not accounted for within a conventional MC approach. This limits the applicability of the method to amorphous or polycrystalline solids where these effects are supposed to be negligible.

\section{SIMULATION RESULTS}

In order to analyze the quality of the proposed scattering model and the associated simulation procedure, we compare here the simulation results with experimental data. These refer mainly to transmission and/or backscattering measurements on thin films; but we shall also present results for backscattering from a thick sample coated with a thin layer of different composition.

Figures 4 and 5 correspond to transmission experiments where an electron beam impinges on thin aluminum films of different thicknesses. The direction of the incident beam is along the normal to the surface of the sample. Angular distributions of transmitted electrons are shown in Fig. 4; the experimental and simulated distributions have been normalized to the same area. The agreement between the data and the numerical results is seen to be satisfactory. Energy distributions of transmitted electrons are presented in Fig. 5. All distributions have been normalized to the same area. Again the simulation leads to good agreement with the experiment.

The results of a MC simulation by Shimizu et al..$^{5}$ are also included in Fig. 5. Simple simulation procedures similar to the one described in Ref. 5 are becoming very popular because of their easy implementation in a computer. How-
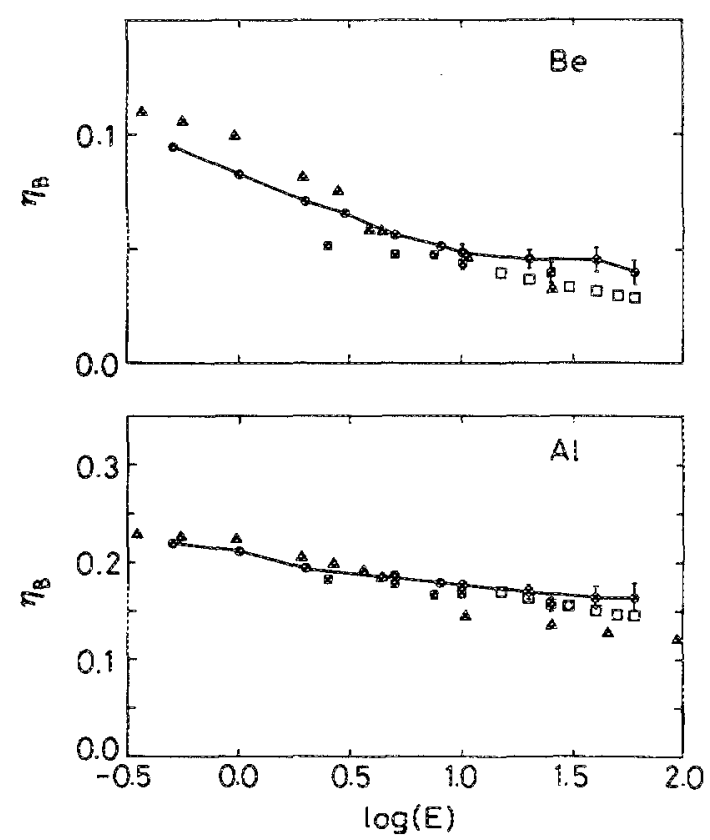

FIG. 9. Energy dependence of the backscattered fraction of electron beams impinging normally on thick samples of beryllium and aluminum. Energies are in keV. Full circles are the results of the present $\mathrm{MC}$ simulations, joined by straight lines for visual aid only; full squares are simulation results obtained from the MCSDA code ( Ref. 15). Other symbols refer to experimental data from Refs. 39 and 40 , and others quoted by Fitting (Ref. 38).

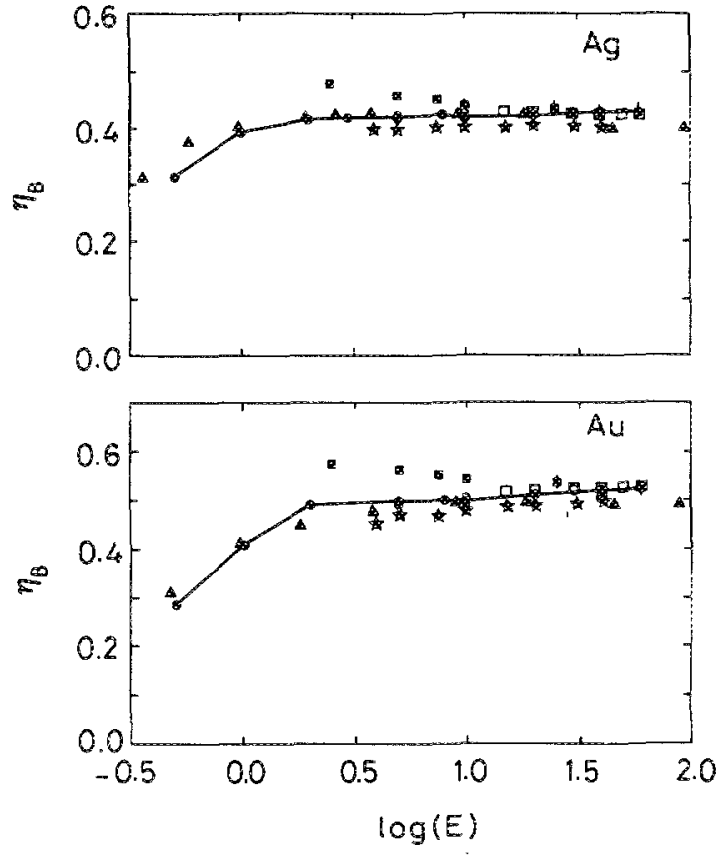

FIG. 10. Energy dependence of the backscattered fraction of electron beams impinging normally on thick samples of silver and gold. Lnergites are in keV, full circles and full squares are simulation results from the present method and from the MCSDA code. ${ }^{15}$ Other symbols refer to experimental data from Refs. 39 and 40 , and others quoted by Firting (Ref. 38 ).

ever, it should be recognized that the approximations adopted in these procedures may lead to important inaccuracies in certain practical applications.

Energy distributions of 2-and 3-keV electrons transmitted through a beryllium layer $80 \mathrm{~nm}$ thick at normal incidence are shown in Fig. 6. This figure contains also the MC results of Liljequist ${ }^{23}$ who used a different GOS model to describe the inelastic collisions and a simple screened Rutherford cross section, depending on an empirical parameter, to simulate the elastic collisions. All the distributions have been normalized to the same area.

The backscattered fraction of a parallel electron beam impinging on a solid film has been measured for a wide range of electron energies, film thicknesses, and compositions. ${ }^{37-41}$ This fraction is of practical importance in scanning electron microscopy and in Auger electron spectroscopy (since a backscattered electron can produce additional contributions to the Auger signal when it returns to the surface region of the sample). Due to its practical importance, a number of semiempirical formulas have been proposed to estimate the backscattered fraction in terms of the beam energy and sample characteristics (see Williamson, Antolak, and Meredith $^{42}$ and references therein). For specific geometrical arrangements, the evaluation of the backscattered fraction demands a suitable $\mathrm{MC}$ simulation.

Backscattered fractions for 20 - and $60-\mathrm{keV}$ electron beams impinging normally on single element slabs are shown in Fig. 7 as functions of the atomic number of the target. MC results, shown as full circles, compare well with the experimental data of Neubert and Rogaschewski, ${ }^{37}$ which have been joined with linear segments for visual aid. 


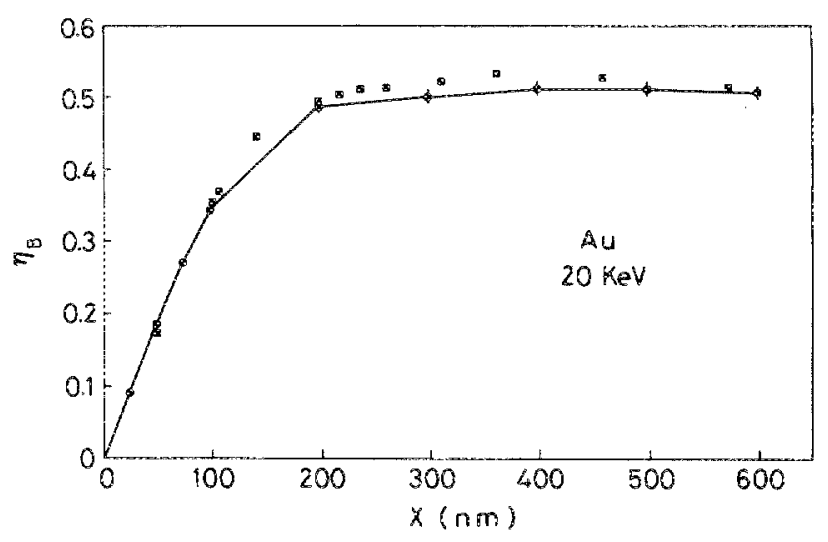

FIG. 11. Backscattered fraction of $20 \mathrm{keV}$ electrons impinging perpendicularly on a gold film as a function of the film thickness. Full circles are the present $\mathrm{MC}$ results, full squares are experimental data from Neubert and Rogaschewski (Ref. 37).

In the experiments, only primary and fast secondary electrons (with kinetic energy larger than $50 \mathrm{eV}$ ) were detected, low-energy secondary electrons were supressed by biasing the sample to $+50 \mathrm{~V}$. In these and the following figures, statistical uncertainties in the simulation results are indicated by error bars (two standard deviations) which in some cases are smaller than the symbol size. The results of $\mathrm{MC}$ simulations without considering the contribution to the backscattered fraction of secondary electrons are also shown in Fig. 7 for $20 \mathrm{keV}$. It is seen that the absolute contribution of secondaries diminishes as the atomic number decreases.

The dependence of the backscattered fraction on the direction of the incident beam is shown in Fig. 8 for aluminum: and uranium and $20 \mathrm{keV}$ electron energy. The angle $\theta_{\text {inc }}$ is that formed by the incident beam and the normal to the sample surface. The lines correspond again to the experimental data of Neubert and Rogaschewsk ${ }^{37}$ joined by linear segments. The full triangles correspond to the simulation results for uranium neglecting the contribution of secondary electrons.

The dependence of the backscattered fraction of normally incident beams on the energy is shown in Figs. 9 and 10 for beryllium, aluminum, silver, and gold. In these figures the $\mathrm{MC}$ results from the present procedure are indicated by full circles joined by linear-segments for visual aid only. Results obtained from the MCSDA code ${ }^{14,15}$ are shown as full squares. This code uses a simple simulation algorithm based on the Born approximation for elastic scattering and the continuous slowing down approximation with an artificial distribution of energy losses to account for straggling. Comparison of the MCSDA and the present simulation results reveals the failure of the Born approximation for low electron energies, which is more pronounced for large atomic numbers, and confirms the validity of the MCSDA backscattered fraction for high electron energies.

The backscattered fraction of a $20-\mathrm{keV}$ electron beam impinging normally on a thirs gold film as a function of its thickness is shown in Fig. 11. The backscattered fraction for aluminum films and for an electron beam of the same energy is given in Fig. 12 (lower set of data). The upper set of data

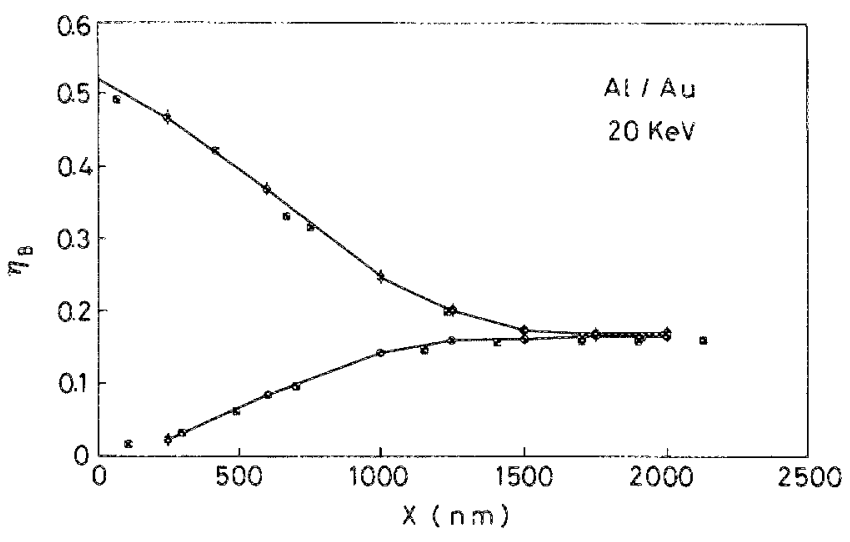

FIG. 12, Backscattered fraction 20-keV electrons impinging perpendicularly on an aluminum thin layer (lower set of data) and on a thick gold slab coated with a thin aluminum layer (upper set of data) as a function of the aluminum layer thickness. Other details are the same as in Fig. 10. Experimental data are from Refs. 37 and 41

corresponds to the backscattered fraction for a thick gold sample coated with an aluminum layer. The thickness dependence is correctly reproduced.

\section{CONCLUSIONS}

We have described a comparatively simple model which gives a reasonable description of electron multiple scattering for energies ranging from a few hundred $\mathrm{eV}$ to several tens of $\mathrm{keV}$. This scattering model has been adopted as the basis of a detailed MC simulation method. Simulation results for transmission and backseattering experiments have been presented and compared with experimental data for different elements and electron energies. Such a MC procedure can be efficiently used to simulate the experimental conditions encountered in surface electron spectroscopy. A study of the effect of elastic scattering in quantitative $\mathrm{x}$-ray photoelectron spectroscopy using the present simulation method is currently in progress.

\section{ACKNOWLEDGMENTS}

We are indebted to Dr. R. Neubert and Dr. S. Rogaschewski for providing us with their experimental results in numerical form. This work has been partially supported by the Spanish CICYT, Grant No. PB86-0589.

1S. Ichimura and R. Shimizu, Surf. Sci. 112, 386 (1981).

${ }^{2}$ A. Jablonski, M. Ebel, and H. Ebel, J. Electron Spectrose. Reiat. Phenom. 40, 125(1986).

${ }^{3}$ D. Liliequist, in Scanning Electron Microscopy, edited by O. Johari (SEM, AMF O'Hare, Chicago, IL, 1983), Vol. III, g. 997.

4I. R. MeDonald, A. M. Lanki, and C.F.G. Delaney, J. Phys. D 4, 1210 (1971).

${ }^{5}$ R. Shimizu, Y. Kataoka, T. Matsukawa, T. Ikuta, K. Murata, and H. Hashimoto, J. Phys. D 8, 820 (1975).

${ }^{\prime} F$. Salvat and J. Parellada, J. Phys. D 17, 185 (1984).

${ }^{7}$ H. J. Fitting and J. Reinhardt, Phys. Status Solidi A 88, 245 (1985).

${ }^{8}$ S. Ichimura, M. Aratama, and R. Shimizu, J. Appl. Phys. 51, 2853 (1980). ${ }^{9}$ M. Kotera, K. Murata, and K. Nagami, J. Appl. Phys. 52, 997 (1985).

${ }^{10}$ A. F. Akkermar and A. L. Gibrekhternan, Nucl. Knstrum. Methods B 6 , 
$496(1985)$.

${ }^{11}$ A. J. Green and R. C. J. Leckey, J. Phys. D 9, 2123 (1976).

${ }^{12}$ D. Liljequist, F. Salvat, R. Mayol, and J. D. Martinez, J. Appl. Phys. 65, 2431 (1989).

${ }^{13}$ F. Salvat, R. Mayol, and J. D. Martínez, J. Phys. B 20, 6597 (1987).

1.7. Salvat and J. Parellada, J. Phys. D 17, 1545 (1984).

${ }^{15}$ F. Salvat, J. D. Martínez, R. Mayol, and J. Parellada, Comput. Phys. Commun. 42, 93 (1986).

in D. Liljequist, J. Phys. D 11, 839 (1978).

${ }^{17}$ M. Gryzinski, Phys. Rev. 138, A336 (1965).

${ }^{1 k}$ R. Shimizu, Y. Kataoka, T. Ikuta, T. Koshikawa, and H. Hashinoto, I. Phys. D 9, 101 (1976).

"C. Dejardin-Horgues, J. P. Ganachaud, and M. Cailler, J. Phys. C9, L633 (1976).

20)A. Desaivo, A. Parisini, and R. Rosa, J. Phys. D 17, 2455 (1984).

${ }^{21}$ D. Liljequist, J. Phys. D 16, 1567 (1983).

${ }^{72}$ F. Salvat, J. D. Martinez, R. Mayol, and J. Parellada, J. Phys. D 18, 299 (1985).

2.D. Liljequist, . Appl. Phys. 57, 657 (1985).

${ }^{24}$ N. F. Mott and H. S. W. Massey, The Theory of A tomic Collisions (Oxford University, Oxford, 1965).

${ }^{25}$ F. Salvat, J. D. Martínez, R. Mayol, and J. Parellada, Phys. Rev. A 36, 467 (1987).

${ }^{2}$ M. Inokuti, Rev. Mod. Phys. 43, 297 (1971).
${ }^{27}$ U. Fano and J. W. Cooper, Rev. Mod. Phys. 49, 441 (1968).

${ }^{2.8}$ J. Lindhard and X. Dan. Vidensk. Selsk., Mat.-Fys. Medd. 28, No. 8 (1954).

${ }^{29} \mathrm{~J}$. Lindhard and A. Wincher, K. Dan. Vidensk. Selsk., Mat.-Fys. Medd. 34, No. 4 (1964).

${ }^{30}$ R. H. Ritchic, Phys. Rev. 14, 644 (1959).

${ }^{31}$ D. R. Penn, Phys. Rev. B 35, 472 (1987).

${ }^{32}$ J. C. Ashley, C. J. Tung, and R. H. Ritchie, Surf. Sci. 81,409 (1979).

${ }^{33}$ C. J. Tung, J. C. Ashley, and R. H. Ritchie, Surf. Sci. 81, 427 (1979)

${ }^{34}$ S. Kirkpatrick and E. P. Stoll, J. Comput. Phys. 40, 517 (1981).

${ }^{35}$ M. H. Kalos and P. A. Whitlock, Monte Carlo Methods (Wiley, New York, 1986), Vol. I.

${ }^{36}$ R. N. Thornas, Ph.D. thesis, Cambridge University, 1961.

${ }^{37}$ R. Neubert and S. Rogaschewski, J. Phys. D 17, 2439 (1984).

${ }^{38}$ H. J. Fitting, Phys. Status Solidi A 26, 525 (1974).

${ }^{39} \mathrm{H}$. E. Bishop, in Electron Beam Interactions with Solids, edited by D. F. K yser, D. E. Newbury, H. Niedrig, and R. Shimizu (SEM, AMF OMare, Chicago, IL, 1984), p. 19 .

${ }^{4}$ H. J. Hunger and L. Kuchler, Phys. Status Solidi A 56, K45 (1979).

${ }^{4}$ R. Neubert and S. Rogaschewski, Phys. Status Solidi A 59, 35 (1980) and 87, $451(1985)$.

${ }^{42}$ H. J. Fitting, J. Phys. D \&, 1480 (1975).

${ }^{43}$ W. Williamson Jr., A. J. Antolak, and R. J. Meredith, J. Appl. Phys. 61, $4612(1987)$. 